\title{
Aportes de la aplicación de la tomografía eléctrica y del georadar en la investigación del deslizamiento de Lidecko en los Cárpatos Occidentales, República Checa
}

\author{
Bladimir Alex Cervantes-Ricalde ${ }^{1, *}$ y Tommhy Lervi Cuadros-León ${ }^{2}$ \\ ${ }^{1}$ Institute of Geological Engineering, Faculty of Mining and Geology, VŠB - Technical University of Ostrava, \\ Av. 17, Listopadu 15/2172, 70833, Ostrava, República Checa. \\ ${ }^{2}$ Department of Geotechnics and Underground Engineering, Faculty of Civil Engineering, VŠB - Technical University of Ostrava, \\ Av. Ludvíka Podéště 1875/17, 70833, Ostrava, República Checa. \\ *cervantesrba@hotmail.com
}

\section{RESUMEN}

Este trabajo se centra en los aportes de la aplicación de mediciones geofísicas utilizando el método de la tomografía de resistividad eléctrica y el radar de penetración terrestre o georadar en la metodología de investigación de los movimientos de laderas, presentando como caso de estudio el deslizamiento de Lidecko, localizado en los Cárpatos Occidentales Exteriores, ubicados en la parte oriental de la República Checa. Se trata de un deslizamiento complejo lento ( $2 \mathrm{~m} / \mathrm{año})$ el cual es frecuente en las estructuras geológicas denominadas Flysch que son típicas de los Cárpatos eslovaco-moravos.

Este artículo muestra las ventajas y beneficios del uso estos dos métodos geoeléctricos en este tipo de investigaciones, en comparación con los métodos geofísicos tradicionales (métodos sísmicos y geoeléctricos) y presenta un procesamiento preciso de los datos medidos en el campo para realizar su análisis e interpretación objetiva. Los resultados obtenidos proporcionan una nueva información sobre el cambio de la estructura interna de la masa rocosa, la geometría y las dimensiones de las áreas afectadas.

Palabras clave: métodos geofísicos; tomografía de resistividad eléctrica; georadar; deslizamientos.

\begin{abstract}
This work is the result of a series of investigations to optimize the methodology of geophysical measurements using the electrical resistivity tomography method and ground penetrating radar in territories affected by slope deformations. Case study and application area presented was, in this paper, selected on the Outer Western Carpathians (Czech Republic).

This paper shows the application of these methods in the research of a landslide typical in the Slovak-Moravian Carpathians. Geologically these mountain ranges are composed mainly of flysch sediment.

The purpose of this paper is to 1) show the advantages and benefits of the relatively new geoelectric methods for the examination of slope deformation, 2) compare them with the standard and traditional
\end{abstract}

geophysical methods and with other geotechnical studies, and 3) present accurate processing of measured data in the field for the appropriate analysis and objective interpretation of the results.

Key words: geophysical methods; electrical resistivity tomography; ground penetrating radar; landslide.

\section{INTRODUCCIÓN}

En la utilización de métodos geofísicos para la exploración e investigación de las deformaciones de laderas se puede observar un rápido desarrollo técnico (Grandjean et al., 2006; Gutiérrez et al., 2010) con la aplicación y selección de diferentes métodos de trabajo (Aracil Ávila et al., 2005; Naudet et al., 2008) e instrumentación (Zerathe y Lebourg, 2012; Heincke et al., 2010). Actualmente, además de los métodos geofísicos tradicionales como sondeos eléctricos verticales, calicatas eléctricas o métodos sísmicos, se han incluido otros métodos geofísicos como la tomografía de resistividad eléctrica (en adelante ERT) (Perrone et al., 2014), el georadar (en adelante GPR) y la tomografía sísmica (Samyn et al., 2012; Le Roux et al., 2011; Heincke et al., 2010; De Bari et al., 2011) para determinar la susceptibilidad y riesgo a deslave de la estructura rocosa de una manera más objetiva y cualitativa (Gutiérrez et al., 2010; Chao et al., 2008).

Sobre el área de los Cárpatos Occidentales Exteriores, ubicados en la República Checa, hay una serie de publicaciones dedicadas a la investigación de movimientos de ladera que ejemplifican una amplia gama de aplicaciones de diversos métodos geofísicos, especialmente de los métodos ERT y tomografía sísmica en combinación con métodos geofísicos convencionales (Baroň y Krejčí, 2005; Blaha y Duras, 2013; Poul et al., 2010).

En este artículo son presentados y discutidos los resultados y los aportes obtenidos de la aplicación de los métodos geofísicos ERT y GPR en el área del deslizamiento de Lidecko (República Checa), en un periodo comprendido entre el año 2011 y 2014, centrándose principalmente en los resultados obtenidos en la parte de transporte y acumulación del deslizamiento. 


\section{CASO DE ESTUDIO}

El área de aplicación de estos dos métodos ERT y GPR está ubicada en los montes Cárpatos Occidentales Exteriores en la República Checa, situada en la subprovincia de los Cárpatos eslovaco-moravos en las tierras altas de Vizovice al Sureste de la República Checa en la región Vsetin (Figura 1).

En esta región se han registrado y analizado una serie de procesos de ladera, de acuerdo al grado de inestabilidad, diferenciándolos en procesos activos (determinados por la velocidad del movimiento presente, tipo de movimiento y la naturaleza de los materiales inestables) e inactivos (en terrenos estables y potencialmente inestables) (CGS, 2017).

Desde el punto de vista geomorfológico, el deslizamiento refleja un alto grado de pendiente y una depresión alargada en el eje del movimiento. Se han detectado bloques de arenisca que están separados por una serie de grietas en la dirección Suroeste a Noreste, aproximadamente perpendicular a la dirección del movimiento. La inclinación media de la pendiente es de $25^{\circ}$, siendo en algunos lugares hasta $40^{\circ}$ (Figura 2). La presencia de la roca arenisca resistente a la erosión provoca una elevación relativa en la zona de hasta $260 \mathrm{~m}$ en $500 \mathrm{~m}$ de longitud.

Desde el punto de vista geológico regional, el deslizamiento se encuentra en la unidad geológica Raca del Paleógeno Magura. Las capas precuaternarias pertenecen a las Formaciones Solan y a los Miembros Luhacovice, donde rocas arenisca y arcosa del Paleoceno y Eoceno se intercalan con la presencia de lutitas limosas y grauvaca. La roca arenisca es de color gris claro hasta marrón con granos de tamaño medio hasta grueso, siendo en algunos sectores conglomerados con una matriz arcillosa calcárea (Baroň, 2004). La cubierta cuaternaria en el área de interés es deluvial representada por sedimentos no cementados muy heterogéneos, en algunos lugares en base de arena y grava. En otras partes se trata de fragmentos rocosos sedimentarios arenosos (Figura 1).

Desde el punto de vista hidrogeológico, estas formaciones precuaternarias tienen una permeabilidad por fisuras que se atribuyen principalmente a las capas degradadas del subsuelo. El agua subterránea está relacionada con los sedimentos cuaternarios deluviales y sedimentos clásticos no cementados (grava, arena) como colectores de permeabilidad intrínseca.

Desde el punto de vista tipológico, este movimiento de ladera estudiado es clasificado como un deslizamiento complejo, situado sobre un antiguo deslizamiento inactivo (Ryšávka y Skopal, 2008; Baroň, 2004). En la Figura 3 se observan las partes del deslizamiento, donde la parte superior es traslacional, mientras que la parte de acumulación (parte inferior) es traslacional en las capas superficiales y rotacional en las capas profundas relacionado probablemente con el antiguo deslizamiento inactivo (Baroň, 2002). La superficie del deslizamiento activo está fuertemente dañada. En la superficie de la parte de acumulación se observan lutitas limosas, grauvaca y bloques de roca arenisca con dimensiones en algunos casos mayores a cinco metros. La velocidad de movimiento es relativamente lenta en los últimos años, con un promedio de $2 \mathrm{~m}$ /año.

El deslizamiento tiene una forma alargada de dirección Oeste - Este, con una longitud de aproximadamente $350 \mathrm{~m}$ y una anchura media de $40 \mathrm{~m}$; en el área de acumulación la anchura del deslizamiento es de $70 \mathrm{~m}$ y en la parte superior (cabecera) unos $30 \mathrm{~m}$ (Figura 3). En

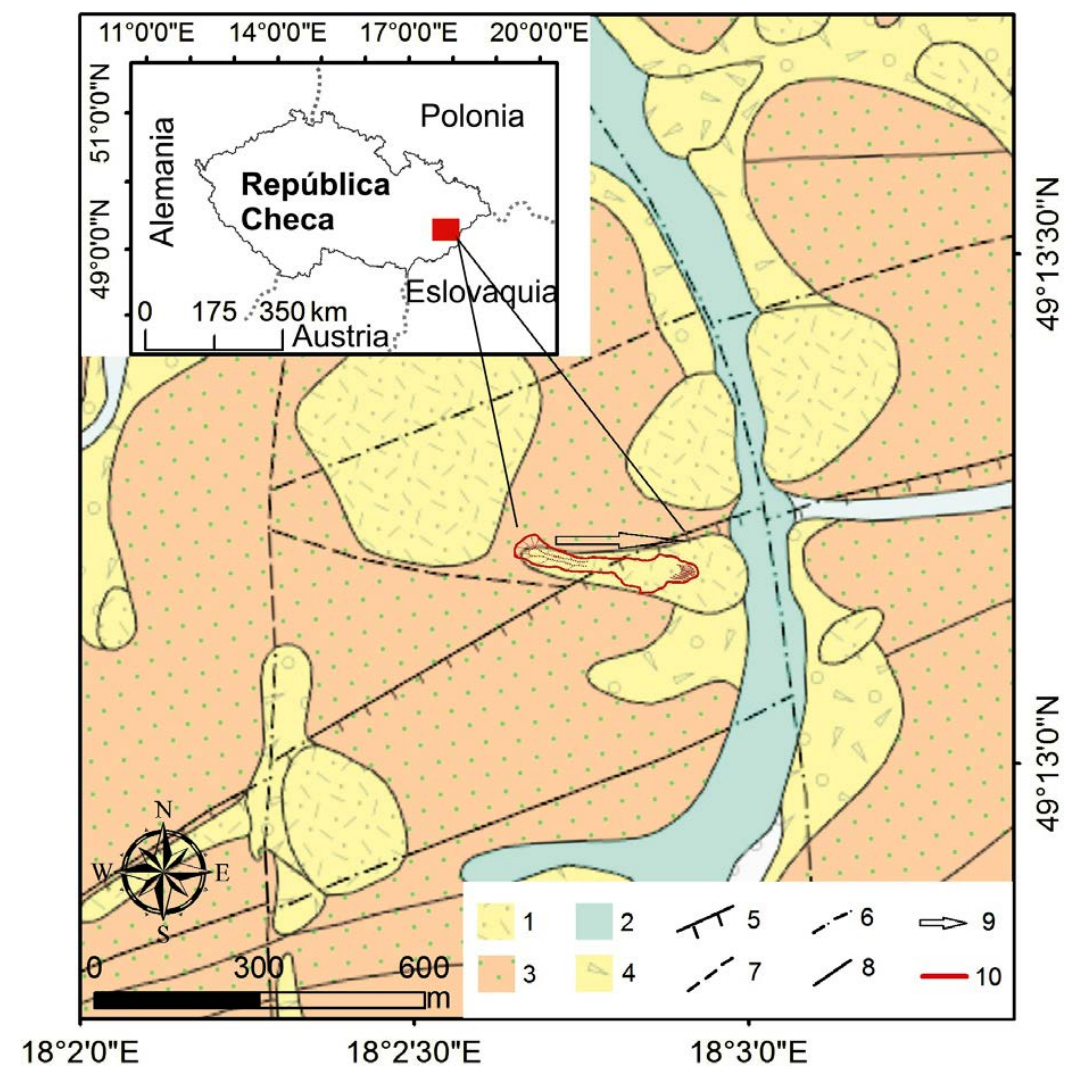

Figura 1. Ubicación del movimiento de la ladera y mapa geológico de la localidad. Simbología: 1, Sedimentos deluviales arenosos arcillosos y grauvacas; 2 , Sedimentos fluviales arena y grava; 3, Areniscas hasta conglomerados y lutitas; 4, Abanicos aluviales gravas; 5, Falla de corrimiento; 6, Falla sepultada; 7, Falla supuesta; 8, Contacto geológico; 9, Dirección de movimiento del deslizamiento; 10, Límites del deslizamiento. Sistema de coordenadas: ETRS 1989 UTM Zone 34N. 


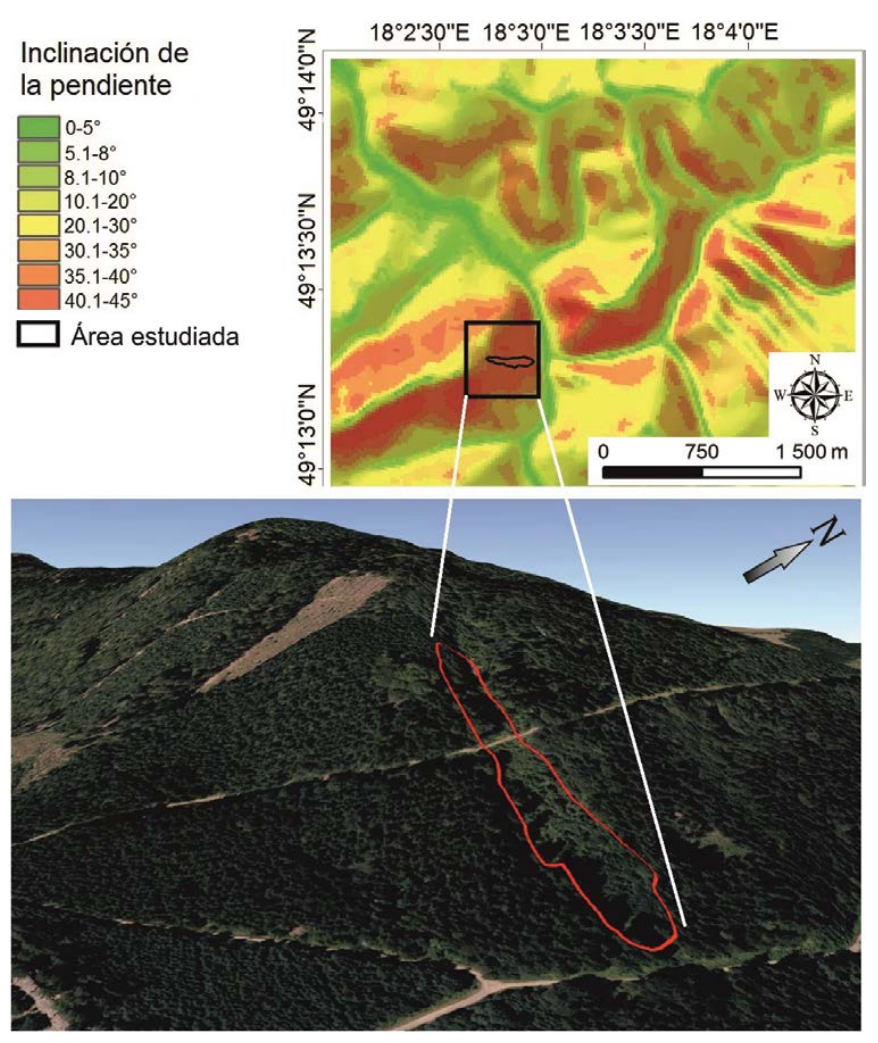

Figura 2. Mapa de inclinación de la pendiente en el área estudiada (ETRS 1989 UTM Zone $34 \mathrm{~N}$ ) y un acercamiento esquemático al deslizamiento en un modelo 3D - línea roja en el modelo (Terrain analysis, 2017).

el área de transporte y acumulación del deslizamiento, el terreno es muy caótico y está cubierto por bloques de rocas junto con restos de árboles arrancados. Estos bloques de roca arenisca macroscópicamente exhiben bajos niveles de erosión.

De acuerdo a los parámetros geológicos e hidrogeológicos y junto a los cambios climáticos (CHMI, 2016) en esta zona se asume que las fuertes precipitaciones del año 1997 fueron el mecanismo de iniciación del deslizamiento, de manera subsecuente se reactivó el movimiento en los meses de mayo y junio de los años 2010 y 2012, producto de la saturación de las areniscas y capas deluviales superficiales no cementadas, creando superficies de rupturas y rajaduras (discontinuidades) a lo largo del movimiento.

Durante el proceso de investigación en esta localidad se efectuaron, en el área de acumulación del deslizamiento, mediciones los métodos de tomografía sísmica y refracción sísmica superficial. En la Figura 4 se puede observar que los valores de velocidad de propagación de las ondas de la refracción sísmica son mayores que los de la tomografía en las capas más superficiales. Esta diferencia se observa hasta la profundidad de $10 \mathrm{~m}$, que probablemente corresponde a las zonas afectadas por el movimiento (zona de falla). Según el análisis de las velocidades de ondas en la interpretación de datos sísmicos, los sedimentos por debajo de esta profundidad tienen velocidades de propagación mucho mayores $(1500-3000 \mathrm{~m} / \mathrm{s})$ que pueden corresponder al basamento de la ladera. Los resultados de estas mediciones sísmicas permitieron obtener a grandes rasgos una estimación general de la morfología del subsuelo de la ladera con base en las propiedades elásticas de los materiales. En base a estos resultados se procedió a la aplicación de las mediciones geoeléctricas para poder especificar el desarrollo del deslizamiento, así como delimitar las diferentes partes del mismo y determinar su litología, considerando que la aplicación de estos métodos (ERT a GPR) es mucho más adecuada para la investigación en una superficie tan dañada como esta.

\section{METODOLOGÍA DE TRABAJO}

\section{El método ERT}

En este trabajo se utilizó como unidad central el resistivímetro ARES (GFInstruments, 2010). El dispositivo de medición utilizado fue Wenner-Schlumberger con una distancia electródica de $4 \mathrm{~m}$. La separación entre los electrodos fue seleccionada de acuerdo al reconocimiento del terreno y al estudio de la documentación existente. La profundidad de investigación deseada fue calculada en base a las Ecuaciones 1 y 2 :

$$
\begin{aligned}
& \mathrm{Pt}=\mathrm{S}_{\text {max }} \cdot \alpha \\
& \mathrm{Pt}=\mathrm{A}_{\text {max }} \cdot \beta
\end{aligned}
$$

donde $\alpha$ y $\beta$ son coeficientes calculados para cada dispositivo elec-

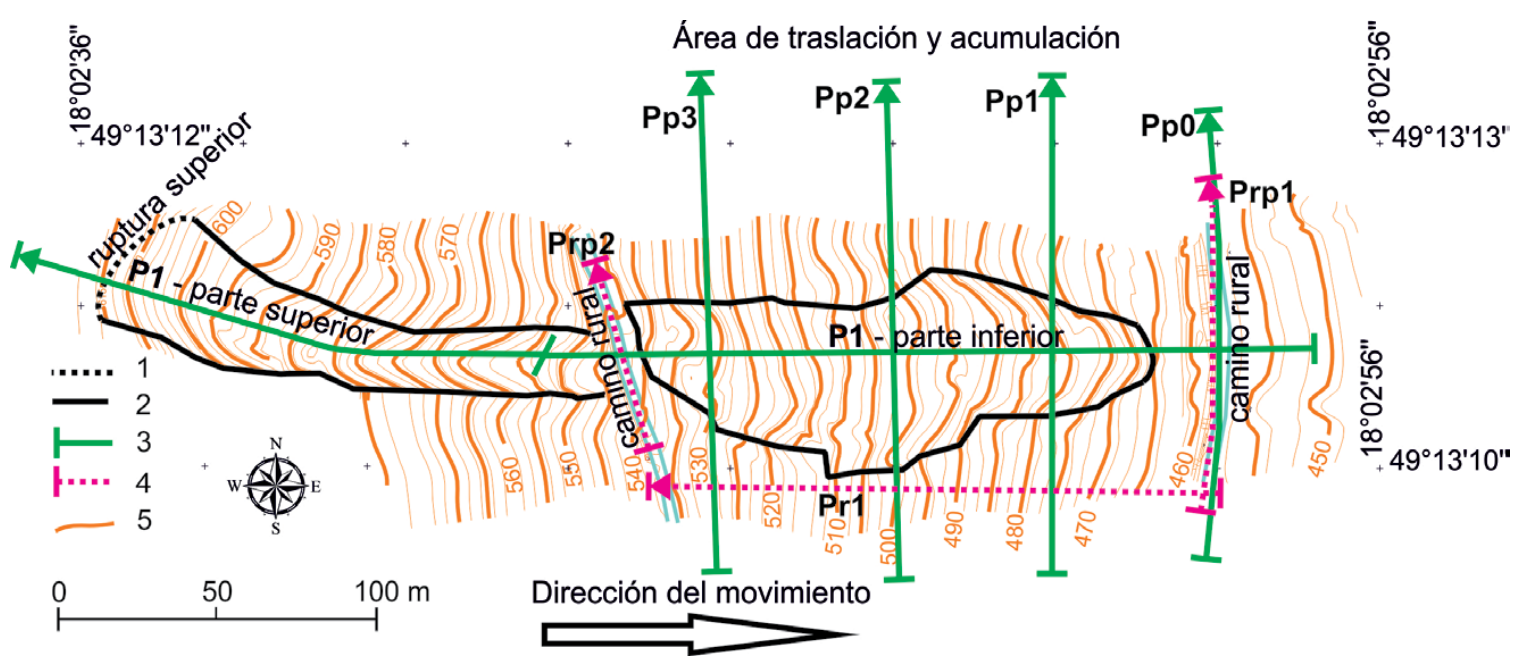

Figura 3. Ilustración del deslizamiento y localización de los perfiles geoeléctricos en el área investigada. Simbología: 1, Cabecera del deslizamiento; 2, Límites del deslizamiento; 3, Perfiles ERT; 4, Perfiles GPR; 5, Curvas de nivel en m s.n.m; Las abreviaciones simbolizan: P1, perfil longitudinal ERT; Pp0, Pp1, Pp2, Pp3, perfiles transversales ERT; Pr1, perfil longitudinal GPR; Prp1 - Prp2, perfiles transversales GPR. 


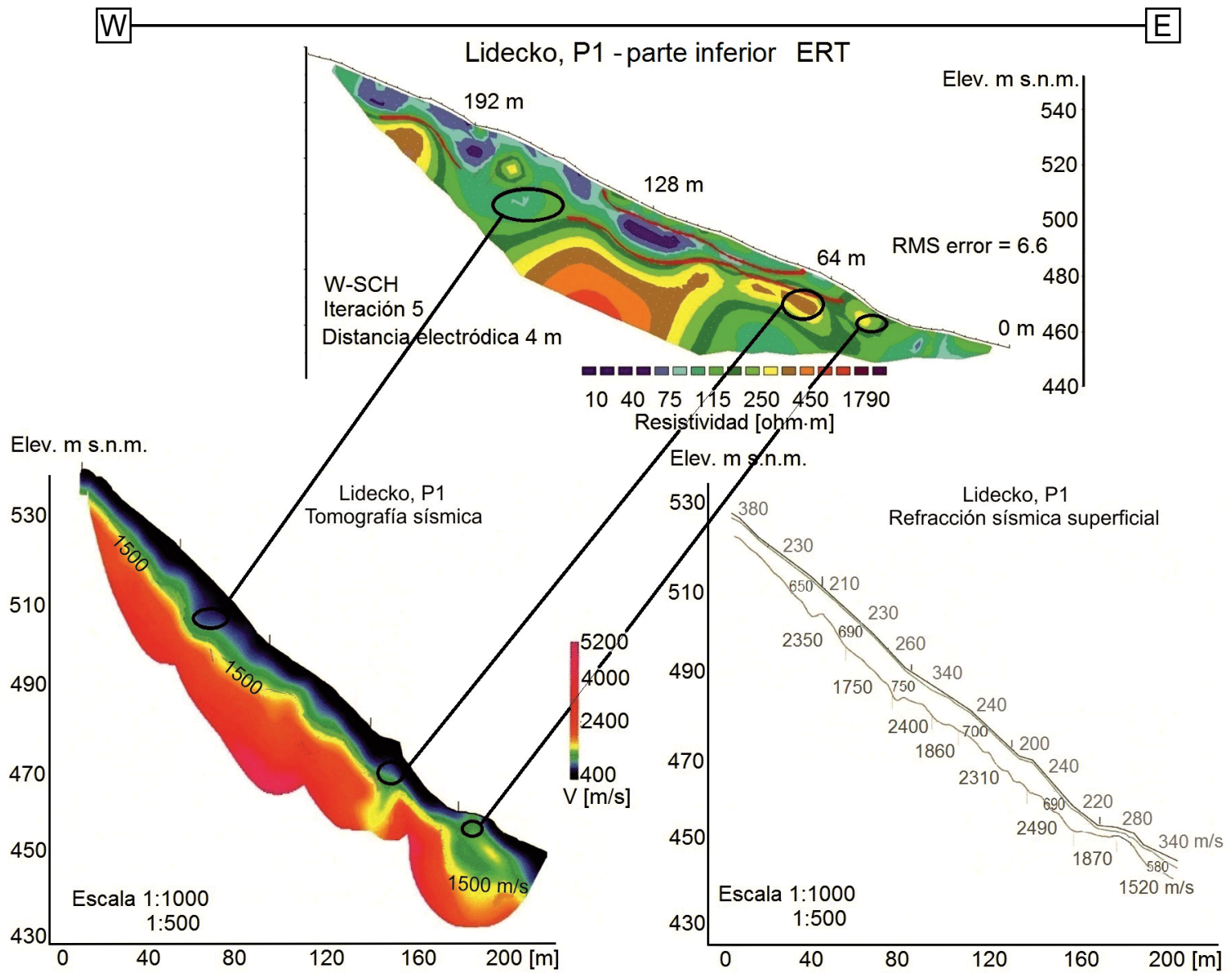

Figura 4. Comparación de la tomografía eléctrica con los métodos sísmicos (tomografía sísmica y refracción sísmica superficial) realizados en el perfil longitudinal P1 (en la parte de acumulación del deslizamiento). Las líneas negras señalan las zonas de concordancia entre los métodos tomográficos. La abreviación W-SCH simboliza el dispositivo Wenner-Schlumberger.

tródico. Los coeficientes para el dispositivo Wenner-Schlumberger se encuentran en Loke, 2014, tab. 2.1. Los valores $\mathrm{S}_{\max }$ y $\mathrm{A}_{\max }$ son la máxima longitud del perfil en medición y la máxima distancia electródica, respectivamente. En esta investigación el valor de $S_{\max }$ fue de 192 metros en el perfil longitudinal P1 (Figura 3) obteniendo una profundidad teórica de 37 metros. En el caso de los perfiles transversales Pp0 - Pp3 (Figura 3) el valor de $S_{\max }$ fue 156 metros calculando una profundidad teórica de 30 metros.

Este cálculo teórico de la profundidad asume que la medición tiene lugar en un entorno homogéneo, por lo que sólo se pueden utilizar como una guía aproximada al momento de planificar la investigación. La profundidad real interpretable obtenida en el perfil longitudinal P1 fue de 33 metros y en los perfiles transversales de 25 metros.

El procesamiento de los datos obtenidos por el método ERT fue realizado en el programa RES2DINV ver. 3.59 (Geotomo Software, 2010).

El tratamiento de los datos obtenidos en el terreno se basa principalmente en el arreglo y/o eliminación de los datos medidos. Por este medio son eliminados los puntos con resistividades claramente erróneas en comparación con los puntos vecinos. Después de esto se realizó el proceso de iteración con un máximo cinco iteraciones (Loke, 2014). Otro factor importante en el proceso de inversión es la corrección topográfica del perfil. En el estudio de los movimientos de ladera puede un relieve accidentado afectar significativamente el modelo resultante.

\section{Método GPR}

En este método se utilizó el georadar RAMAC/GPR (Mala GeoScience, 2004). Este georadar está constituido por un sistema de dos antenas no apantalladas unidas por cables reforzados de fibra óptica en una configuración lineal. En esta disposición de operación, conocida también como common-offset, las antenas mantienen una separación constante entre ellas de 4 metros, moviéndose en la misma dirección a lo largo del perfil medido. La aplicación de este georadar en la investigación de una superficie tan accidentada como esta proporciona un mejor contacto con el suelo y realiza mediciones continuas. $\mathrm{La}$ frecuencia central de operación de la antena utilizada fue de $50 \mathrm{MHz}$, permitiendo obtener teóricamente una alta penetración de 20 a 30 metros con una resolución de 50 centímetros (Mala GeoScience, 2006).

En el caso de las mediciones realizadas con el método GPR el procesamiento de los datos adquiridos se llevó a cabo con el uso del programa RadExplorer 1.4 distribuido por la compañía sueca Mala Geoscience (DECO-Geophysical, 2005).

Las transformaciones básicas más importantes realizadas en el procesamiento de datos de los radargramas (Cassidy, 2009; Kazunori et al., 2012) fueron la eliminación de ruido (señales) de fondo para mejorar la imagen resultante, la corrección de las reflexiones superficiales (corrección de tiempo cero), la migración de las reflexiones para la localización de las estructuras investigadas y el arreglo topográfico de los perfiles para evitar una distorsión significativa en los radargramas. Los 
parámetros más importantes tomados en cuenta antes de la medición fueron el ajuste de la frecuencia de muestreo con un valor aproximado de 10 veces mayor al de la frecuencia de la antena (Mala GeoScience, 2007), el seleccionado de las ventanas de tiempo, la consideración de la velocidad estimada de las ondas en el medio estudiado, el contador de pulsos e intervalos y la correcta orientación de las antenas hacia la estructura buscada.

\section{MEDICIÓN E INTERPRETACIÓN DE LOS RESULTADOS}

Se realizaron varios perfiles de medición ERT a lo largo del eje del deslizamiento y en dirección perpendicular al movimiento (Figura 3). El perfil longitudinal indicado como P1 se realizó en el eje del movimiento conformado de dos partes con longitudes de $254 \mathrm{~m} \mathrm{y}$ $286 \mathrm{~m}$ (Figura 5). En dirección perpendicular al eje del movimiento se realizaron cuatro secciones transversales $\mathrm{Pp} 0$ - Pp3, donde el perfil transversal Pp0 fue ubicado fuera del área del deslizamiento para realizar una comparación del área afectada con el área estable (Figura 6).

La división del perfil P1 se realizó debido a que la longitud total del deslizamiento es muy grande y a las condiciones muy complicadas del terreno. El propósito de esta medición fue detectar los diferentes contrastes geoeléctricos de la ladera hasta una profundidad de $30 \mathrm{~m}$ en las diferentes partes del deslizamiento (corona o cabecera, las zonas de transporte y de acumulación). Se consiguió de esta forma distinguir las probables superficies de ruptura, discontinuidades en el cuerpo del deslizamiento y las zonas predispuestas a la saturación por el agua (Figura 5). Con base en estos resultados se procedió a la realización de los perfiles transversales en la parte de transporte y acumulación del deslizamiento (marcados en el perfil P1 como Pp0 - Pp3 con un triángulo, ver Figura 5). Se puede observar la similitud de las resistividades en los perfiles transversales ubicados en el cuerpo del deslizamiento y la diferencia con el perfil en el extremo inferior, al pie de la zona inestable. Claramente se pueden delimitar (con base en los significativos contrastes de resistividad) diferentes partes de inestabilidad que son correlacionales con los resultados en el perfil longitudinal P1. En las secciones geoeléctricas interpretadas (Figura 6) se puede distinguir la parte activa del deslizamiento (marcada con una línea roja punteada), la presencia de deformaciones inactivas o fósiles (línea roja) así como las probables zonas de discontinuidad (líneas negras). En la interpretación de las secciones geoeléctricas (Figura 6) también se puede distinguir la presencia de los sedimentos conductores arcillo-limosos (10 - $40 \Omega . \mathrm{m})$, de las areniscas saturadas $(50-200 \Omega . m)$, de las areniscas resistivas (250 - $700 \Omega . \mathrm{m})$ y de las acumulaciones de los depósitos deluviales (500 - $1000 \Omega . m)$.

Después de la interpretación de estos resultados y considerando que se trata de un movimiento lento $(2 \mathrm{~m} / \mathrm{año})$ se procedió a la siguiente etapa de investigación, es decir, realizar un seguimiento de los cambios de resistividad en la parte más inestable del deslizamiento en el periodo 2012 - 2014. Los resultados obtenidos se muestran en la Figura 7. Al comparar las secciones verticales de resistividades efectuadas en intervalos anuales se demuestra que en este período se produjo un desarrollo desfavorable en la superficie de ruptura. Se puede observar una significativa demarcación de la inestabilidad de la ladera a una profundidad de $6-8 \mathrm{~m}$ (línea roja punteada) y de una probable reactivación de la falla fósil entre 15 a $20 \mathrm{~m}$ (línea roja). También se puede constatar que las condiciones climáticas en la realización de las mediciones anuales fueron similares (CHMI, 2016).

Las mediciones GPR en el área de interés se llevaron a cabo en marzo del 2014. Estas mediciones fueron realizadas después de una previa interpretación de los resultados ERT y del seguimiento del movimiento en la zona de acumulación y traslación, para poder establecer los parámetros más adecuados del GPR con la antena de $50 \mathrm{MHz}$ de frecuencia. Se trazaron tres perfiles, uno longitudinal

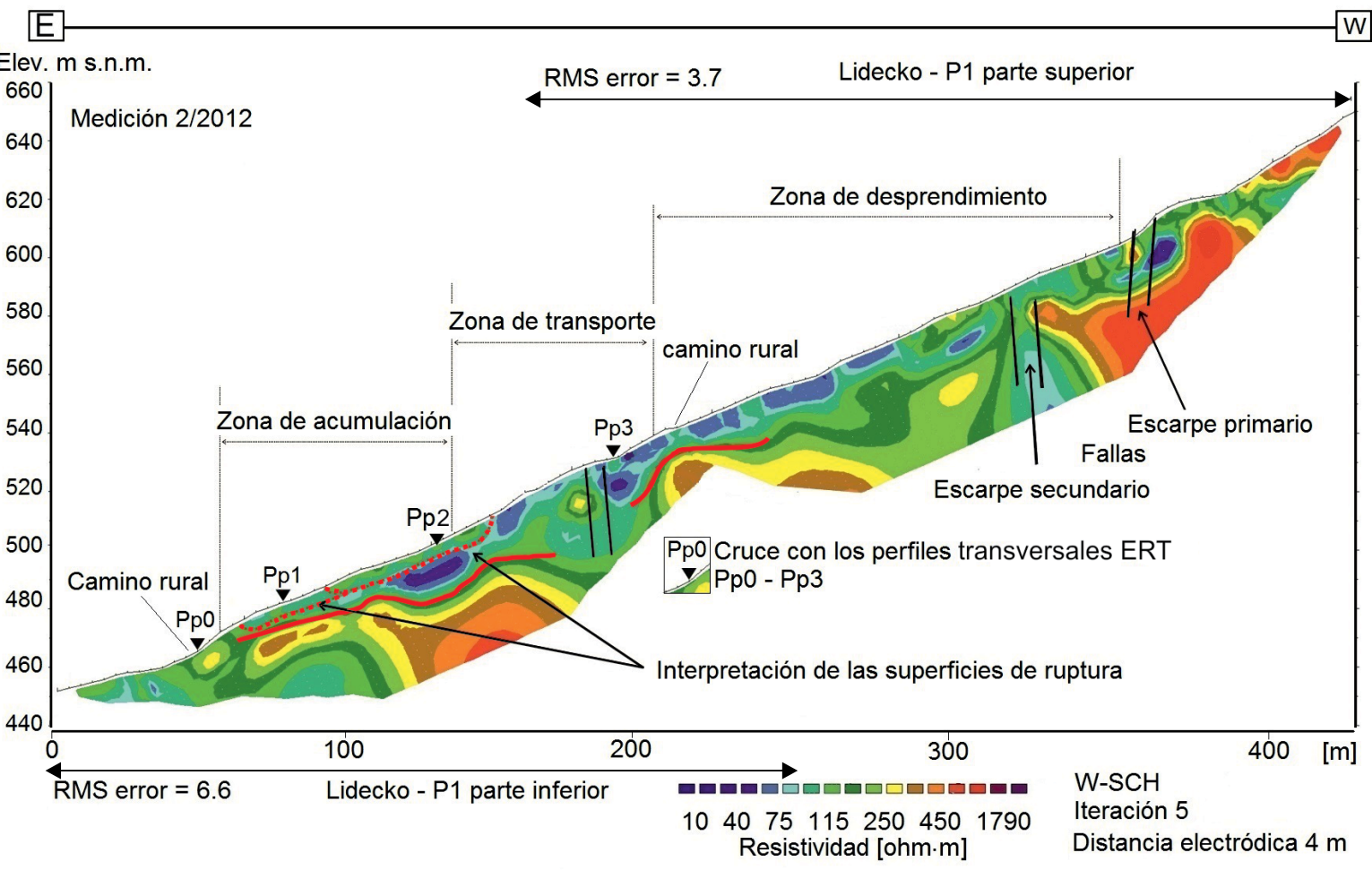

Figura 5. Interpretación del perfil tomográfico eléctrico P1 y la representación de las principales partes del deslizamiento. Las abreviaciones simbolizan: W-SCH, dispositivo Wenner-Schlumberger; Pp0, Pp1, Pp2, Pp3, perfiles transversales ERT. 


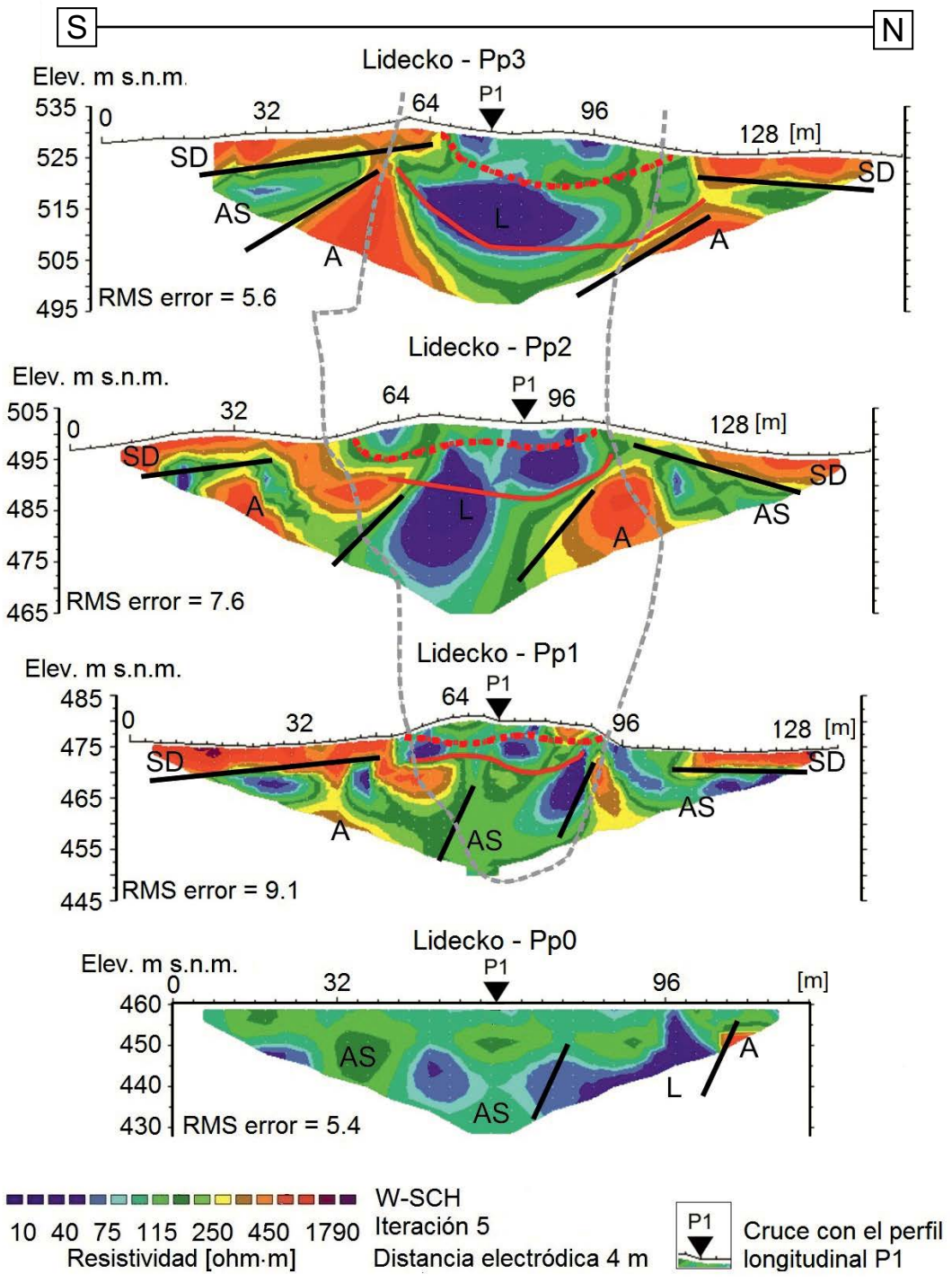

Figura 6. Interpretación de los perfiles tomográficos eléctricos transversales al eje del movimiento. Las abreviaciones simbolizan: W-SCH, Dispositivo Wenner-Schlumberger; A, Areniscas; AS, Areniscas saturadas; L, Lutitas; SD, Depósitos deluviales. Las líneas negras representan el contacto geológico. La línea roja punteada representa la superficie de ruptura activa del deslizamiento. La línea roja entera marca la base del movimiento inactivo y la línea ploma punteada delimita el deslizamiento en la parte de acumulación y transporte. Los perfiles transversales ERT estan simbolizados con las abreviaciones Pp0, Pp1, Pp2, Pp3. paralelo al eje de movimiento Pr1 y dos perpendiculares a este Prp1 y Prp2 (Figura 8). La extensión total de los perfiles fue de 360 metros. El primero con una longitud de 200 metros y los otros dos Prp1 con 100 metros y Prp 2 con 60 metros. Después de repetidas mediciones se establecieron los siguientes parámetros como los más adecuados, la frecuencia de muestreo entre los 500 y $800 \mathrm{MHz}$ con un tiempo de muestreo de $0.5 \mathrm{~s}$ en el perfil longitudinal y $0.3 \mathrm{~s}$ en los transversales. La profundidad máxima de estudio obtenida fue de 28 metros (Figura 8).

El curso de ondas reflejadas en el perfil longitudinal Pr1 impide delimitar con claridad las capas más superficiales del deslizamiento, debido a su baja resolución. Sin embargo, se puede delimitar la profundidad y continuidad de la roca arenisca en determinados segmentos (línea negra en la Figura 8).

En el caso de los perfiles transversales Prp1 y Prp2 la medición fue afectada por la conductividad del subsuelo (saturado con el agua infiltrada de las lluvias). La resolución de estos perfiles muestra la interfaz entre las areniscas secas y areniscas saturadas (limitadas por una línea negra) detectando también la influencia del camino rural en el terreno (línea verde punteada), interpretados en la Figura 8. Con la comparación de estos dos métodos se obtiene la interpretación geofísica de la inestabilidad de la ladera.

\section{RESULTADOS Y DISCUSIÓN}

La utilización del método ERT en esta investigación mostró satisfactoriamente los cambios en la estructura interna del deslizamiento desde el punto de vista de la resistividad eléctrica y su respectiva variabilidad en el espacio-tiempo. Estos cambios se ven afectados directamente por las precipitaciones en las diferentes estaciones del año, así como la cantidad de agua infiltrada que modifica la presión en los poros de los sedimentos, creando de esta forma un contraste físico capaz de ser medido. El análisis de estos cortes geoeléctricos y de los radargramas permite obtener información relativa de la geometría del deslizamiento, como las superficies de ruptura, fisuras internas y/o posibles zonas de presión en el pie del deslizamiento (elipse negra en la Figura 9), así como también la litología del espacio afectado (Figura 9).

Las mediciones realizadas con estos métodos y con esta metodología (técnicas) presentan resultados estáticos, que se aplican al momento de la medición, sin mostrar signos de la dinámica del movimiento. Con la aplicación de las mediciones repetitivas (método ERT) se puede conseguir información de los cambios físicos en el tiempo, pero que no necesariamente están relacionados con la dinámica del deslizamiento. Por lo tanto, la interpretación de los resultados sirve como una guía para 


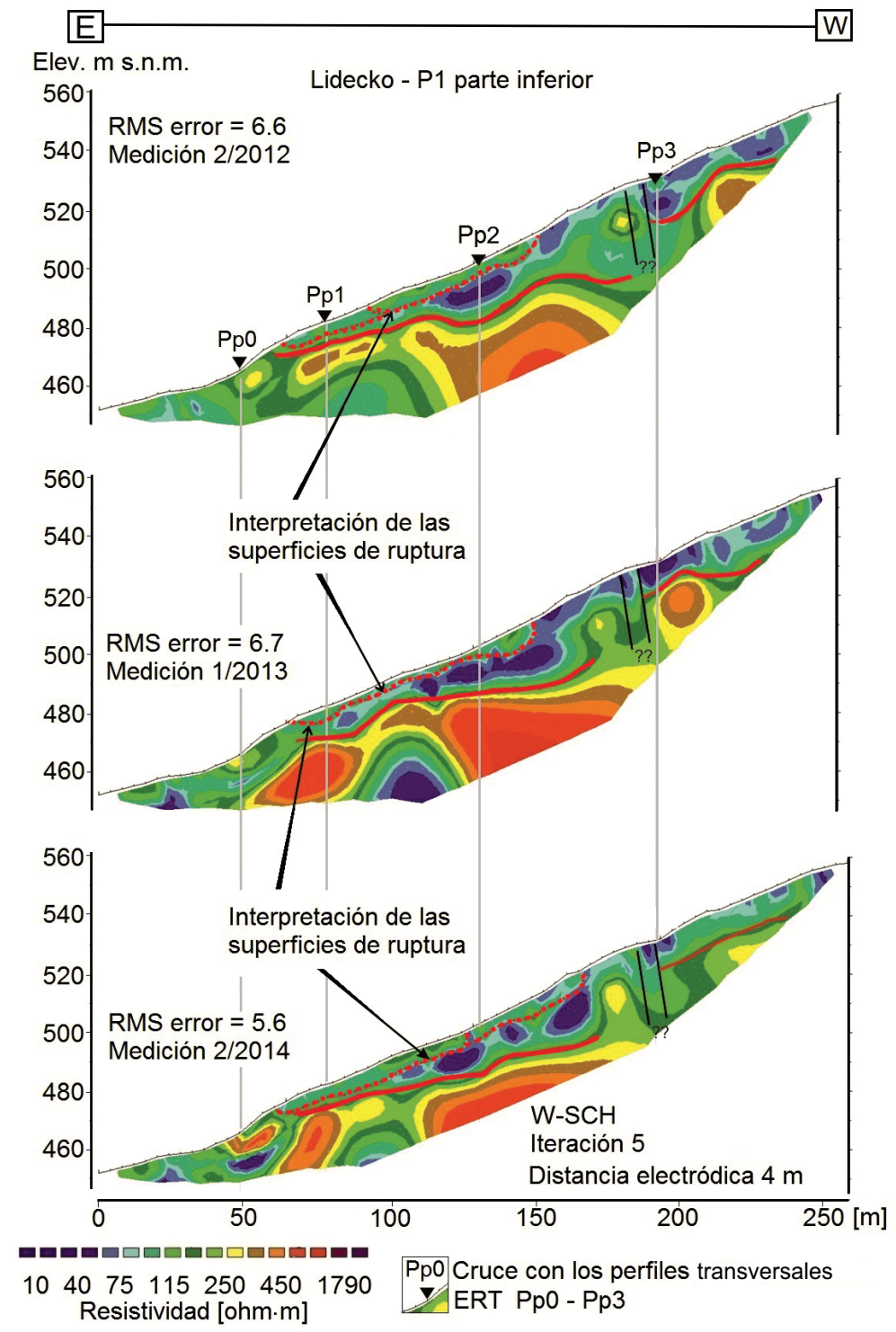

Figura 7. Comparación de los resultados de medición del perfil tomográfico eléctrico P1 efectuados en intervalos anuales. Las líneas negras representan una posible falla. La línea roja punteada representa la superficie de ruptura activa del deslizamiento. La línea roja entera marca la base del movimiento inactivo. Las abreviaciones simbolizan: W-SCH, dispositivo Wenner-Schlumberger; P1, perfil longitudinal ERT; Pp0, Pp1, Pp2 Pp3, perfiles transversales ERT.

la delimitación del espacio afectado por este fenómeno y la consecuente realización de una adecuada clasificación para proceder a los trabajos de mitigación y su respectivo saneamiento.

El proceso de interpretación de los datos por el método ERT dependerá mucho de los parámetros técnicos de medición del dispositivo utilizado, la separación entre los electrodos, la fijación de los electrodos en el suelo, así como también de las condiciones naturales del entorno geológico.

Las mediciones realizadas con el uso de la antena de $50 \mathrm{MHz}$ no apantallada dan resultados satisfactorios, inclusive en estas condiciones muy complicadas, donde se puede observar que el rango de profundidad (penetración) es atenuado por la presencia de limolitas y areniscas saturadas por el agua.

Desde un punto de vista cuantitativo, la utilización de estos dos métodos desplaza a los métodos geoeléctricos tradicionales como el sondeo eléctrico vertical y calicatas eléctricas a un segundo plano. En el caso de medir un perfil de $300 \mathrm{~m}$ de longitud con un rango de profun- didad de $30 \mathrm{~m}$, usando el sondeo eléctrico vertical (suponiendo que se realicen unos 11 perfiles) se obtienen aproximadamente 200 puntos de medición. En el caso de medir este mismo perfil utilizando el método de calicata eléctrica se obtienen cerca de 300 puntos de medición. Con la aplicación del método ERT en este mismo perfil se obtienen más de 800 puntos de medición con el dispositivo Wenner-Schlumberger. De este modo queda claro que los resultados ERT en el estudio de los movimientos de laderas son más resolutivos y objetivos.

\section{CONCLUSIONES}

Los resultados obtenidos con estos dos métodos en esta localidad permiten completar y especificar las condiciones geológicas, así como observar el desarrollo del deslizamiento, deducidos de la primera etapa de investigación con las mediciones geofísicas (métodos sísmicos), particularmente en la delimitación de las superficies de ruptura. Es 

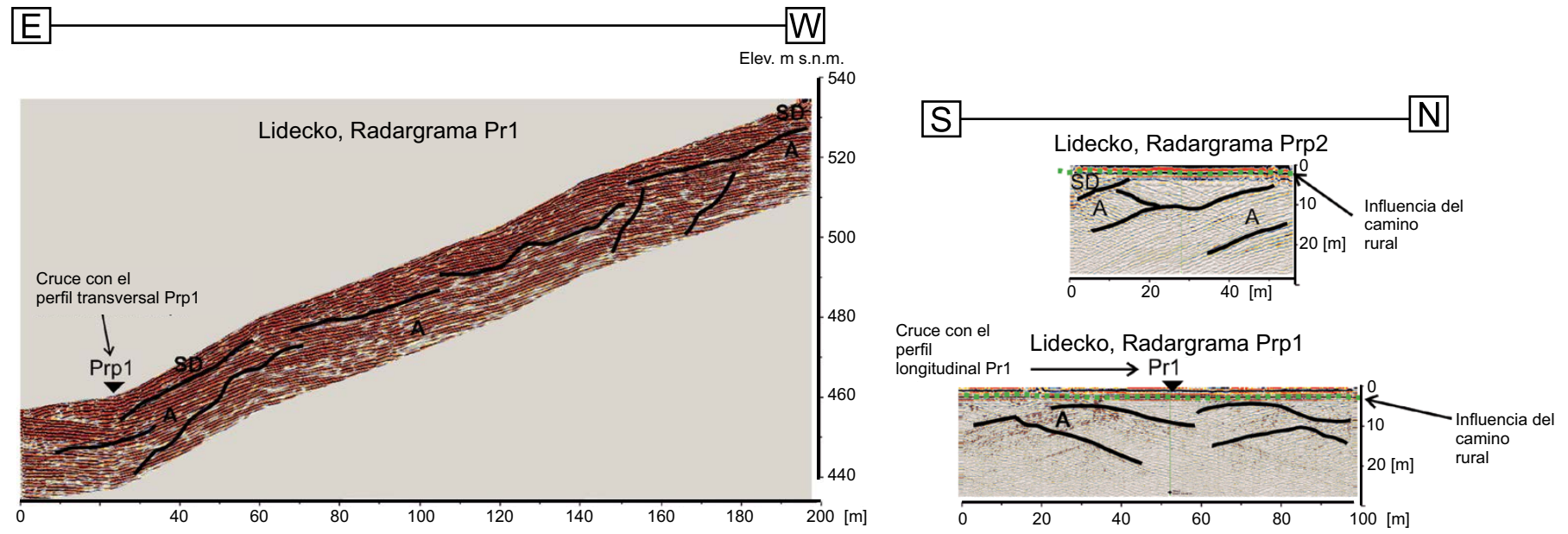

Figura 8. Interpretación de los radargramas obtenidos sobre el deslizamiento utilizando un georadar no apantallado de $50 \mathrm{MHz}$ y corregidos por topografía. Las líneas negras muestran los planos de reflexiones con las rocas areniscas. Las abreviaciones simbolizan: A, Areniscas; SD, Sedimentos deluviales; Pr1, perfil longitudinal GPR; Prp1, Prp2, perfiles transversales GPR.

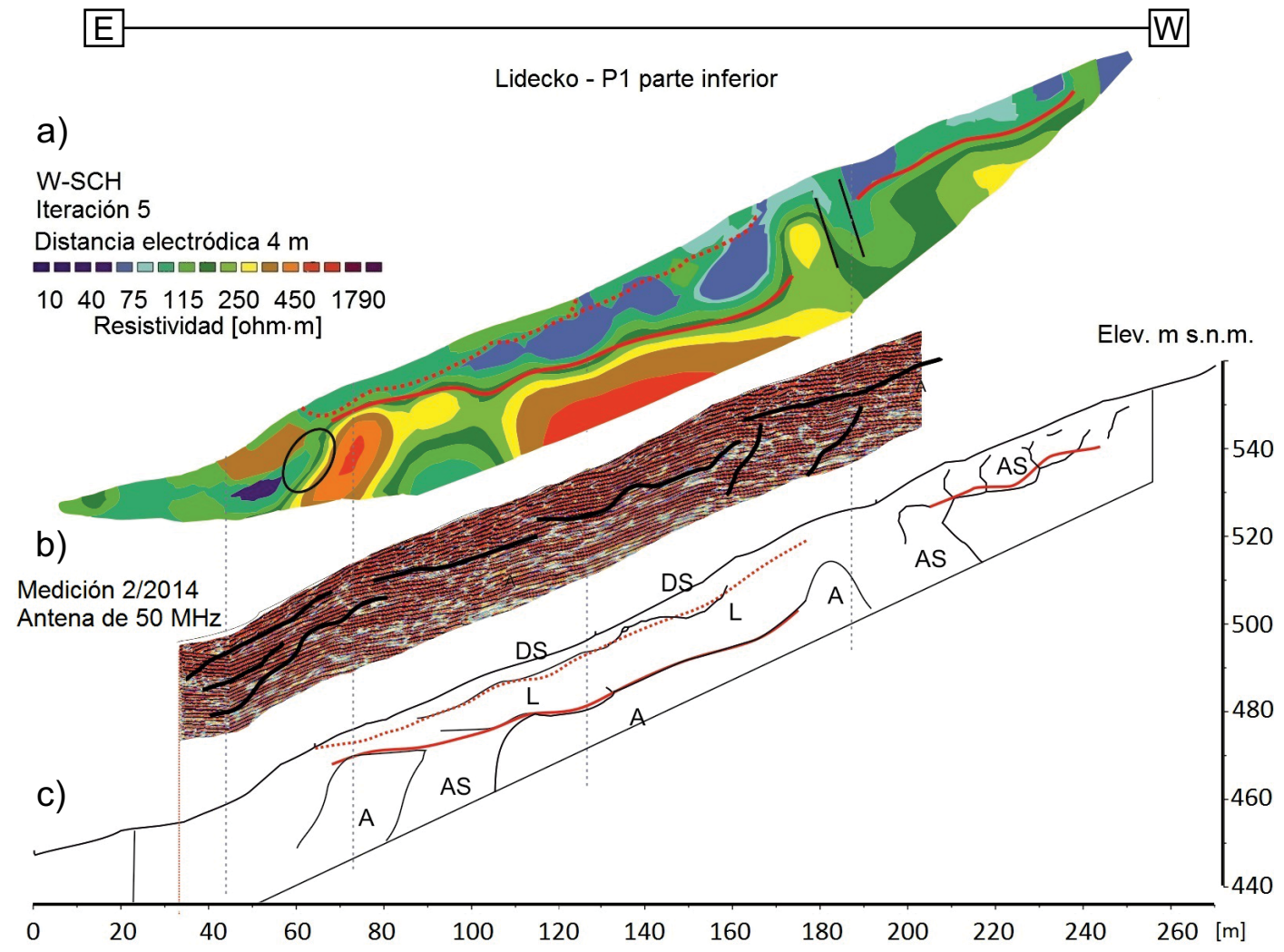

Figura 9. Interpretación geofísica del deslizamiento en la parte inferior del perfil P1. a) Interpretación del perfil ERT. b) Interpretación del radargrama. c) Interpretación geofísica del deslizamiento en su parte de traslación y acumulación. Las abreviaciones simbolizan: W-SCH, Dispositivo Wenner-Schlumberger; A, Areniscas; AS, Areniscas saturadas; L, Lutitas; DS, Deposito deluvial. La línea roja entera y punteada representan la superficie de ruptura activa y la base inactiva del deslizamiento. La línea negra detecta las reflexiones con las rocas areniscas. La elipse negra representa posibles zonas de presión en el pie del deslizamiento. 
importante mencionar que las condiciones del terreno fueron poco apropiadas para la realización de cualquier método de investigación in situ, lo cual afectó las mediciones, desde la colocación de los electrodos hasta la elección optima de los perfiles geofísicos.

Otro de los aportes más importantes en la utilización de estos dos métodos es la obtención de datos de una forma rápida y en tiempo real, lo que contribuye a una interpretación más objetiva de los resultados obtenidos.

Para el método ERT, con el fin de no perder la sensibilidad de medición de las resistividades (López Hidalgo et al., 2012; Loke, 2014) debido a las complicadas condiciones del terreno, se realizaron perfiles que no superaron los 200 metros de longitud $\left(S_{\max }\right)$ en pendientes superiores a $30^{\circ}$. La distancia electródica de cuatro metros permitió obtener una mayor profundidad de medición. Gracias a ello, las mediciones no fueron afectadas por las capas superficiales extremadamente dañadas del deslizamiento (en algunos lugares hasta una profundidad de $1.5 \mathrm{~m}$ ). Los perfiles largos se realizaron con la técnica conocida como roll-along, que permitió cubrir distancias superiores a la longitud del dispositivo $\left(\mathrm{S}_{\max }\right)$ manteniendo el rango de profundidad propuesto constante.

La realización de un único perfil ERT y/o GPR, o su combinación, brinda solamente un resultado de muchos que se pueden obtener, debido a la anisotropia característica de los suelos. Por ello es recomendable realizar varios perfiles en diferentes direcciones con respecto al eje de movimiento, si las condiciones del terreno son favorables.

\section{REFERENCIAS}

Aracil-Ávila, E., Porres-Benito, J.A., Espinosa-González, A.B., Maruri-Brouard, U., Vallés-Iriso, J., García Castillo, L., Ibáñez-García, S., 2005, Aplicación de la tomografía eléctrica para la caracterización de un deslizamiento de ladera en un vertedero, en Corominas, J., Alonso, E., Romana, M., Hürlimann, M. (eds.) VI Simposio Nacional sobre Taludes y Laderas Inestables: Valencia, España, Universidad Politécnica de Valencia y UPC, 8 pp.

Baroň I., 2002, The changes of a flow landslide shape after two years, en Rybar J., Stemberk J., Wagner P. (eds.), Landslides Proceedings of the $1^{\text {st }}$ European Conference on Landslides: Prague, Czech Republic, Swets \& Zeitlinger, Lisse, 341-342.

Baroň, I., 2004, Deep-seated slope failure at the Kopce Hill (flysch belt of Western Carpathians): Results of geomorphic and geophysical survey: Geological research in Moravia and Silesia, 11, 82-87.

Baroň, I., Krejčí, O., 2005, Slope instability hazard evaluation and landslide sites monitoring in the Flysch Western Carpathians (Czech Republic), en Proceedings of the Conference "Mass movement hazard in various environments": Polish Geological Institute Special Papers, 20, 9-13.

Blaha, P., Duras, R., 2013, Seismic measurements in engineering geology monitoring of rock mass: Exploration Geophysics, Remote Sensing and Environment Journal, 1, 10-31.

Cassidy, N.J., 2009, Electrical and magnetic properties of rocks, soils, and fluids, en Harry, J. (ed.), Ground Penetrating Radar Theory and Applications: Elsevier Science, 41-72.

CGS, 2017, Ground instabilities (en línea), Map applications: Czech Geological Survey, <http://mapy.geology.cz/svahove_nestability>, acceso libre, consulta, 25 de febrero de 2017.

Chao, L., Yang, C., Liu, H., Wen, K-L., Wang, Z., Chen, Y., 2008, A Study of the hydrogeological environment of the lishan landslide area using resistivity image profiling and borehole data: Engineering Geology, 98, 3-4.

CHMI, 2016, Historical data - Meteorology and Climatology (en línea),: Czech Hydrometeorological Institute, <http://portal.chmi.cz/>, acceso libre, consulta: 10 de diciembre de 2016.

De Bari, C., Lapenna, V., Perrone, A., Puglisi, C., Sdao, F., 2011, Digital photogrammetric analysis and electrical resistivity tomography for investigating the Picerno landslide (Basilicata region, southern Italy): Geomorphology, 133 (1), 34-46.
DECO-Geophysical, 2005, RadExplorer 1.4, The software for GPR data processing and interpretation - User Manual: Moscu, Rusia, DECOGeophysical Co. Ltd., 92 pp.

Geotomo Software, 2010, RES2DINV ver. 3.59, Rapid 2-D Resistivity \& IP inversion using the least-squares method, Geoelectrical Imaging 2D \& 3D: Malaysia, Geotomo Software, User manual, $151 \mathrm{pp}$.

GFInstruments, 2010, Ares - Automatic Resistivity System, User manual: GFInstruments Geophysical equipment and services: Czech Republic, v. 5.0, 103 pp.

Grandjean, G., Pennetier, C., Bitri, A., Meric, O., Malet, J., 2006, Caractérisation de la structure interne et de l'état hydrique de glissements argilo-marneux par tomographie géophysique: l'exemple du glissement-coulée de SuperSauze (Alpes du Sud, France): Comptes Rendus Geoscience, 338 (9), 587-595.

Gutiérrez, F., Soldati, M., Audemard, F., Bălteanu, D., 2010, Recent advances in landslide investigation: Issues and perspectives: Geomorphology, 124 (3-4), 95-101.

Heincke, B., Günther, T., Dalsegg, E., Steinar Ronning, J., Venvik Ganerod, G., Elvebakk, H., 2010, Combined three-dimensional electric and seismic tomography study on the Åknes rockslide in western Norway: Journal of Applied Geophysics, 70(4), 292-306.

Kazunori, T., Igel, J., Holger, P., Kuroda, S., 2012, Basics and application of ground-penetrating radar as a tool for monitoring irrigation process, en Kumar, M. (ed.), Problems, perspectives and challenges of agricultural water management: InTech, Chapters published, 156-180.

Le Roux, O., Jongmans, D., Kasperski, J., Schwartz, S., Potherat, P., Lebrouc, V., Lagabrielle, R., MERIC O., 2011, Deep geophysical investigation of the large Séchilienne landslide (Western Alps, France) and calibration with geological data: Engineering Geology, 120 (1-4), 18-31.

Loke, M.H., 2014, Tutorial: 2-D and 3-D electrical imaging surveys: Malaysia, Geotomo software, $180 \mathrm{pp}$.

López Hidalgo, A.A., Loke, M.H., Fanton Gerardo, O., Cara Rubí, E., 2012, Técnicas prácticas para investigacion de resistividad en dos y tres dimensiones (tomografía electrica 2d y 3d): San Juan, Argentina, Instituto Geofísico Sismológico, 36 pp.

Mala GeoScience, 2004, RAMAC RTC v. 1.0, Operating Manual: Swede, Mala GeoScience, Operating manual, $13 \mathrm{pp}$.

Mala GeoScience, 2006, RAMAC/GPR v. 1.1, Antennas - Operating Manual, Sweden, Mala GeoScience, Operating manual, $28 \mathrm{pp}$.

Mala GeoScience, 2007, Mala XV Monitor - Operating Manual, Mala GeoScience, Sweden, Operating manual v. 1.5, 42 pp.

Naudet, V., Lazzari, M., Perrone, A., Loperte, A., Piscitelli, S., Lapenna, V., 2008, Integrated geophysical and geomorphological approach to investigate the snowmelt-triggered landslide of Bosco Piccolo village (Basilicata, southern Italy): Engineering Geology, 98 (3-4), 156-167.

Perrone, A., Lapenna, V., Piscitelli, S., 2014, Electrical resistivity tomography technique for landslide investigation: A review: Earth-Science Reviews, $135,65-82$.

Poul, I., Krejčí, O., Hubatka, F., 2010, Underestimated aspect of the building foundations - paleo-landslides in Neogenne marine clays in the north part of Brno city, Geological research in Moravia and Silesia, 17, 195-198.

Ryšávka, J. Skopal, R., 2008, Lidečko-I etapa sanačních prací, geotechnický průzkum: Ostrava, Czech Republic, UNIGEO, Reporte técnico, 30 pp.

Samyn, K., Travelletti, J., Bitri, A., Grandjean, G., Malet, J.P., 2012, Characterization of a landslide geometry using 3D seismic refraction traveltime tomography: The La Valette landslide case history: Journal of Applied Geophysics, 86, 120-132.

Terrain analysis, 2017, Geoportal access to map products and services (en línea), en State Administration of Land Surveying and Cadastre: Czech Republic, $<$ http://ags.cuzk.cz/dmr/>, acceso libre, consultado 2 de marzo de 2017.

Zerathe, S., Lebourg, T., 2012, Evolution stages of large deep-seated landslides at the front of a subalpine meridional chain (Maritime-Alps, France), Geomorphology, 138 (1), 390-403.

Manuscrito recibido: febrero 14, 2017

Manuscrito corregido recibido: mayo 3, 2017

Manuscrito aceptado: mayo 5, 2017 